\title{
Comparison of macular pigment optical density between phakic and pseudophakic eyes.
}

\author{
Burcu Polat Gültekin*, Ayten Bulut, Neslihan Bilen, Defne Kalayci \\ Department of Ophthalmology, Numune Training and Research Hospital, Ankara, Turkey
}

\begin{abstract}
Background: Cataract surgery has been an independent risk factor in the development and progression of age-related macular degeneration. Understanding the relationship between cataract surgery and Macular Pigment Optical Density (MPOD) will be helpful for the prevention of this degeneration during follow-up. The present study is aimed to evaluate effectiveness of lens status and postoperative period over MPOD.

Method: Patients were divided into 2 groups, including phakic cases that have transparent natural lens and pseudophakic cases that had undergone cataract surgery at least a year prior. MPOD values were measured by a heterochromatic flicker photometric method and assessed for each group.

Results: Sixty eyes of 31 cases were enrolled in the study. As group-1 included 30 eyes of 15 phakic cases; group 2 included 30 eyes of 16 pseudophakic cases. The mean MPOD values were $0.572 \pm 128$ and 0.549 \pm 219 in groups 1 and 2, respectively. The mean MPOD values of phakic and pseudophakic patients were statistically similar $(p>0.05)$. However, the MPOD values and duration of postoperative period of the patients in group-2 showed a significant negative correlation $(r=-0.443, p<0.05)$.

Conclusion: The outcome of macular pigment optical density was similar in both phakic and pseudophakic eyes. Although an inverse correlation exists between the duration of the postoperative period after cataract surgery and MPOD values, our results supports that cataract surgery has no potential effects on macular degeneration at least done for a year.
\end{abstract}

Keywords: Heterochromatic flicker technique, Pseudophakia, Macular degeneration, Macular pigment optical density. Accepted on February 17, 2018

\section{Introduction}

Macular pigments are mostly concerted in macula foveal region and consist of retinal carotenoids, mesozeaxanthin, lutein and zeaxanthin [1,2]. They are located in photoreceptor axons in the foveola, internal and external macula plexiform layers. These substances absorb blue-light in the inner side of retina and do function as free-radical-scavengers in the photoreceptors [3,4]. Macular pigments have properties of light absorbing with maximum absorption at $460 \mathrm{~nm}$ [4]. Therefore, macular pigments help reduce some critical processes in the retina, which can result in macula-based disorders such as Agerelated-Macular Degeneration (AMD) [5]. Retinal damage extra to contact with oxidative stress emerges medically as AMD. This disease is the most regularly encountered reason behind vision damage in patients above the age of 65 years in the developed countries [6]. Additionally, various reports showed that macular pigment optical density (MPOD) levels were lower than those discovered in healthies. Macular pigment is considered to have a defensive role against development of AMD [4]. Thus, dedication of the factors impacting on MPOD has kept its importance. Even though surgery was proven to be an unbiased risk element for emerging table of AMD [7,8], some magazines have reported in contrast results [5,9]. Because higher contact with light decrease MPOD in eye which has been subject to surgery, a reduction of MPOD was likely to become as time passes [10-12]. Analysis of cataract surgery and MPOD together may be beneficial for preventing AMD during the follow-up. Different ways of calculating MPOD were used, such as motion-detection photometry, Raman spectrometry, fundusreflectance spectroscopy, Heterochromatic-Flickering Photometry (HFP), and auto-fluorescence spectrometry. Dave et al. described how MPOD can be reliably measured in the presence of cataract with flicker photometry [13]. Whereas Sasamoto et al. reported that cataracts affect MPOD measurements by auto fluorescence spectrometry [14]. We aimed to assess influence of surgery and the duration of the postoperative-period compared with native lenses on MPOD measurements by using flicker technique.

\section{Methods}

\section{Study population}

All patients were separated into 2 lens groups based on the status of lens, after a detailed ophthalmologic examination. The first group had transparent natural lens, which we called as 
phakic cases. The second group we called as pseudophakic cases who had a surgery at least 1 year prior with the best corrected visual-acuity of being equal and higher than 0.5 , based the Snellen-chart. The study was performed between the dates of March and September 2016. Exclusion criteria were existence of any ocular problem that can damage the retina tissue, lens, or optic nerve (e.g., glaucoma and diabetic retinopathy), smoking, iris with light or colored, and use of micro-nutrition supplement. According to the Helsinki Declaration, all individuals signed the informed consent before the examination. The MPOD were measured for both eyes, using the heterochromatic-flicker-photometric method (MPS II, Electron Technology).

\section{Technique}

Heterochromatic-flicker-photometric method is commonly used psycho-physical solution to check the macular pigment concentration. This highly reproducible, pain-free, noninvasive method which does not need a fundus dilatation, is conformity with the absorption range produced from zeaxanthin and lutein. This system needs blue-light with a maximum level close to macular pigment maximum absorption and green-light as stimulant. The strength of blue-light was fine-tuned to calculate pigment thickness of the analysed area, as non-absorbable green-light was for a reference necessity. The computation of MPOD obtains taking the logarithm of proportion of blue-light intensities in both fovea and perifoveal locations with max and min levels of macular-pigment.

\section{Statistical analysis}

All analyses were conducted by the SPSS v18 software (SPSS Inc., Chicago, IL., USA). An independent sample t-test was used for comparing two groups in terms of MPOD values. Spearman's rank-correlation test was used to analyse the relationship between MPOD and duration of postoperativeperiod of the patients in group 2. A p-value less than 0.05 was accepted as statistically significant.

\section{Results}

The study included 60 eyes of 31 cases. Twenty female $(65 \%)$ and 11 male patients $(35 \%)$ with a mean age of $58.3 \pm 7.4$ (range: 50-77 y) were enrolled. Group 1 included 30 eyes of 15 phakic cases (mean age: $54.6 \pm 4.8$ y); group 2 included 30 eyes of 16 pseudophakic cases (mean age: $61.8 \pm 8.1 \mathrm{y}$ ). There was no statistically significant difference in terms of the ages of 2 groups ( $>0.05)$.

The clinic examinations included the best corrected visualacuity with Snellen chart, biomicroscopy of slit lamp, intraocular pressure and ophthalmoscopy. The mean MPOD values were measured as $0.572 \pm 128$ in group 1 and $0.549 \pm$ 219 in group 2. Although MPOD levels were slightly lower in the pseudophakic than phakic group, this impairment was not significant $(\mathrm{t}=0.488, \mathrm{p}>0.05)$ (Figure 1$)$. In group 2, all cases were with acrylic-intraocular lenses. The postoperative follow- up period of 16 patients that underwent cataract surgery was $2.9 \mathrm{y}$ (ranged 1 to $8 \mathrm{y}$ ).

The correlation between MPOD values and the duration of postoperative period in group 2 showed a significant difference $(\mathrm{r}=-0.443, \mathrm{p}=0.02)$ (Figure 2). MPOD levels significantly decreased, while duration of postoperative period indicated an increase.

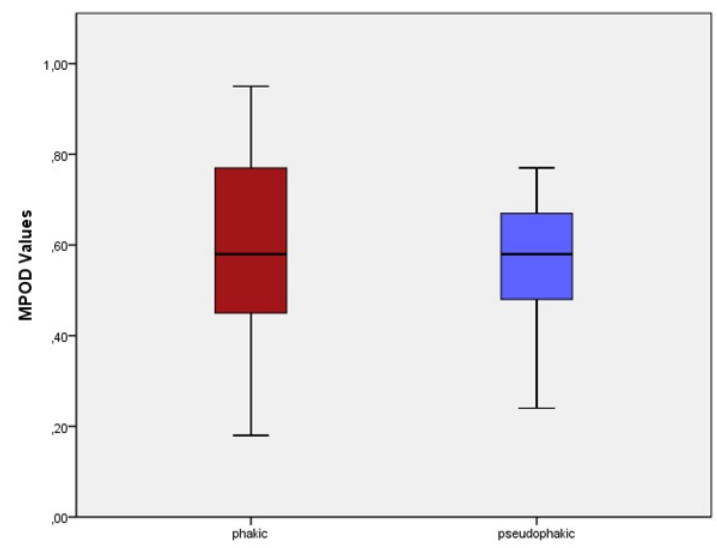

Figure 1. Correlation between MPOD and postoperative period in the patients with cataract surgery.

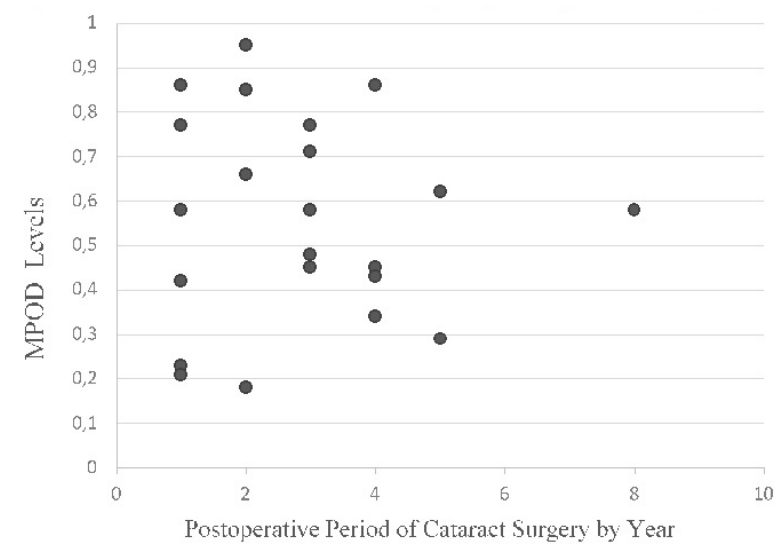

Figure 2. Correlation between MPOD and postoperative period in the patients with cataract surgery.

\section{Discussion}

Recent studies reported different effects of MPOD on the development of AMD. These studies illuminate the value and risks on the perseverance of the factors impacting on the degrees of MPOD [2,8,10,15]. Even though the surgery has been proven to be a risk element for developing AMD, some reports noticed to contrast results [5,9]. In application of surgery, the alternative of the lens with intraocular lens leads to higher blue-light exposure to the retina. Novel reports pointed MPOD measurements out after surgical operations with intraocular lens which blocks blue-light [4]. MPOD and cataract surgery needs to be clarified for illuminating the partnership between surgery and AMD. In the present study, we demonstrated MPOD in conditions of the zoom lens status, 
with the consequences of surgery and duration of postoperative-period on MPOD.

Many studies have reported the relationship between MPOD value and the development of maculopathy [14,16,17]. Many of those were conducted by using auto-fluorescence, HFP, FRS, and SLO. Dave et al. described how MPOD can be reliably measured in the presence of cataract with flicker photometry [13]. Sasamoto et al. reported that cataracts affect MPOD measurements by auto fluorescence spectrometry [14]. As we used HFP method, we assumed that the MPOD measurements were reliable in phakic cases. Whether a clear Intraocular Lens (IOL) or yellow-tinted IOL is used in pseudophakic eyes is also influential in the long-term. Excessive light exposure with clear IOLs is associated with a decrease in macular pigment levels [18]. Our results also indicated lower macular pigment values in eyes with standard intraocular lenses, as the retina is exposed to higher level bluelight.

Despite some reports and epidemiological data, the risk of macular degeneration after cataract extraction should be considered during the patients' evaluation [9]. Determination of changes in MPOD values can help to address this controversial theme $[11,19]$. As well-known, human crystalline lens replacement with surgical approach exposes retinal area to oxidative-stress [20]. Hence, surgery has been accepted as a potential risk in developing and progressing to AMD. According to the fact that there is a negative correlation between MPOD levels and postoperative period, it may be proposed that cataract surgery can be an important long-term factor in maculopathy induction. As reported by Komar et al. [2], the absolute MPOD values were lower after cataract extraction than existed before extraction surgery. Demirel et al. also established lower MPOD levels after surgery and a reverse connection between duration of postoperative-period and MPOD values [10]. Their outcomes are similar to the previous studies reported that MPOD auto-regulation system may not be effective, if retina tissue is exposed to higher level blue-light following the surgery.

A different study evaluated MPOD values in the individuals who were implanted during surgery by commonly used intraocular lens with normal UV filters or blue-light filtering intraocular lens. They reported higher level MPOD in the former group and after a year follow-up [21]. Obena et al. assessed the changes in levels of MPOD following the surgery and noticed that MPOD began decreasing at the end of the year in the commonly used intraocular lens implanted group, when these values started to decrease only at the second year at a lower extent in group of the blue-light filtering lens [15]. In our study, MPOD levels were slightly lower in the pseudophakic than phakic group. But, this impairment was not statistically significant. The postoperative follow-up period of 16 patients that underwent cataract surgery was $2.9 \mathrm{y}$, with ranged 1 to $8 \mathrm{y}$. In correlation analyse, MPOD levels significantly decreased, while duration of postoperative period indicated increasing.

The present study has a few limitations. The first is having a small sample size to make a generalization covering whole society. Second, different age groups of phakic and pseudophakic patients should be studied. Third, the present study was performed in only one institution with limited cases, and the data collected cannot be representative of all other health centers. Fourth, postoperative follow-up period could be longer. Wide- scale-randomized prospective studies are needed with longer follow-up to gain more enlightened results on this research area.

In conclusion, our study has indicated that there is a significant correlation between values of macular pigment density and postoperative period of the cataract surgery. MPOD values showed decrease in long-term follow-up of the patients with pseudophakia. Larger prospective studies with longer followup periods are required to properly address this topic.

\section{Conflict of Interest}

All authors declared that they had no proprietary interests, financial support or conflicts of interest in the present submission.

\section{References}

1. Junghans A, Sies H, Stahl W. Macular pigments lutein and zeaxanthin as blue light filters studied in liposomes. Arc Biochem Biophys 2001; 391: 160-164.

2. Komar B, Rauscher FG, Wiedemann R, Dawczynski J. Macular pigment optical density measurements by onewavelength reflection photometry-influence of cataract surgery on the measurement results. Graefes Arch Clin Exp Ophthalmol 2014; 252: 1717-1727.

3. Mellerio J. Light absorption and scatter in the human lens. Vision Res 1971; 11: 129-141.

4. Schwiegerling J. Blue light-filtering intraocular lenses and scotopic sensitivity. J Cataract Refract Surg 2009; 35: 2032.

5. Sutter FKP, Menghini M, Barthelmes D, Fleischhauer JC, Kurz-Levin MM, Bosch MM. Is pseudophakia a risk factor for neovascular age-related macular degeneration? Investigative Opthalmol Vis Sci 2007; 48: 1472.

6. Loane E, Stack J, Beatty S, Nolan JM. Measurement of macular pigment optical density using two different heterochromatic flicker photometers. Curr Eye Res 2007; 32: 555-564.

7. Klein BEK, Howard KP, Lee KE, Iyengar SK, Sivakumaran TA, Klein R. The relationship of cataract and cataract extraction to age-related macular degeneration: the Beaver Dam eye study. Ophthalmology 2012; 119: 1628-1633.

8. Wegner A, Khoramnia R. Cataract is a self-defence reaction to protect the retina from oxidative damage. Med Hypoth 2011; 76: 741-744.

9. Chew EY, Sperduto RD, Milton RC, Clemons TE, Gensler GR, Bressler SB. Risk of advanced age-related macular degeneration after cataract surgery in the age-related eye disease study. Ophthalmology 2009; 116: 297-303.

10. Demirel S, Bilici S, Bat $\ddot{A} \pm$ oglu F, Ozmert E. The effect of age and cataract surgery on macular pigment optic density: 
a cross-sectional, comparative study. Graefes Arch Clin Exp Ophthalmol 2014; 252: 213-218.

11. Krohn DL, Asbell P, Ullian K, Katz S. Relative blue-light extinction in lens nucleus related to age and cataract. Am J Ophthalmol 1981; 91: 598-602.

12. Puell MC, Palomo-Alvarez C, Barrio AR, Gómez-Sanz FJ, Pérez-Carrasco MJ. Relationship between macular pigment and visual acuity in eyes with early age-related macular degeneration. Acta Ophthalmologica 2013; 91: 298-303.

13. Dave V, Narayanan R. Metamorphopsia assessment before and after vitrectomy for macular hole. Investig Opthalmol Vis Sci 2010; 51: 6895.

14. Sasamoto Y, Gomi F, Sawa M, Sakaguchi H, Tsujikawa M, Nishida K. Effect of cataract in evaluation of macular pigment optical density by autofluorescence spectrometry. Investig Ophthalmol Vis Sci 2010; 52: 927-932.

15. Obana A, Gohto Y, Tanito M, Okazaki S, Gellermann W, Bernstein PS. Effect of age and other factors on macular pigment optical density measured with resonance Raman spectroscopy. Graefes Arc Clin Exp Ophthalmol 2014; 252: 1221-1228.

16. Trieschmann M, van Kuijk FJGM, Alexander R, Hermans P, Luthert P, Bird AC. Macular pigment in the human retina: histological evaluation of localization and distribution. Eye 2007; 22: 132-137.

17. Raman R, Rajan R, Biswas S, Vaitheeswaran K, Sharma T. Macular pigment optical density in a south indian population. Investig Opthalmol Vis Sci 2011; 52: 7910.
18. Obana A, Tanito M, Gohto Y, Gellermann W, Okazaki S, Ohira A. Macular pigment changes in pseudophakic eyes quantified with resonance Raman spectroscopy. Ophthalmology 2011; 118: 1852-1858.

19. Liew SHM, Gilbert CE, Spector TD, Mellerio J, Kuijk FJV, Beatty S. Central retinal thickness is positively correlated with macular pigment optical density. Exp Eye Res 2006; 82: 915-920.

20. Brockmann C, Schulz M, Laube T. Transmittance characteristics of ultraviolet and blue-light-filtering intraocular lenses. J Cataract Refract Surg 2008; 34: 1161-1166.

21. Nolan JM, Stack J, OConnell E, Beatty S. The relationships between macular pigment optical density and its constituent carotenoids in diet and serum. Investig Opthalmol Vis Sci 2007; 48: 571.

\section{*Correspondence to}

Burcu Polat Gültekin

Department of Ophthalmology

Numune Training and Research Hospital

Turkey 